\title{
PREVALENACE OF VANCOMYCIN RESISTANT ENTEROCOCCI IN TERTIARY CARE HOSPI- TAL, WESTERN, INDIA
}

\author{
MODI G.B., SONI S.T., PATEL K.J., GOSWAMI H.M. AND VEGAD M.M. \\ Microbiology Department, B.J. Medical College, Ahmedabad- 380016, GJ, India. \\ *Corresponding Author: Email- gaurav1985bj@gmail.com, drsumeetasoni@gmail.com
}

Received: February 27, 2012; Accepted: March 29, 2012

\begin{abstract}
Introduction: Vancomycin Resistant Enterococci (VRE) is fast emerging pathogen causes serious \& life threatening hospital borne infections. The limitation in therapeutic options has resulted in the development of new drugs such as Quinupristin/ Dalfopristin, Linezolid \& Tigecycline.

Objectives: This study signals the emergence of VRE in this hospital and also highlights the importance of screening for VRE in isolated enterococci from various clinical samples.

Materials and Methods: From July 2010 to July 2011, a total of 250 enterococcal isolates were identified and speciated by standard biochemical tests. Antibiotic sensitivity was carried out by disc diffusion test as per CLSI guidelines. Minimum Inhibitory Concentration (MIC) of all the isolates were done by Vancomycin Ezy MICTM Strip ranging from $0.016 \mu \mathrm{g} / \mathrm{ml}$ to $256 \mu \mathrm{g} / \mathrm{ml}$.

Results: Out of the 250 Enterococcus species, $4 \%$ (10) isolates were resistant to Vancomycin by disc diffusion \& Vancomycin MIC test in which 8 were $E$. faecium and 2 were $E$. faecalis. Out of 10 VRE, 7 were resistant and 3 were sensitive to Teicoplanin, so they were of VanA and VanB phenotype respectively. The Vancomycin MIC for seven of these isolates is more than $256 \mu \mathrm{g} / \mathrm{ml}$. $1.2 \%$ showed intermediate resistance and $2.8 \%$ high level resistance to vancomycin by MIC according to CLSI guidelines.

Conclusion: All laboratories should have effective detection methods for Vancomycin resistance, which will be helpful in treatment of VRE, judiciously with drugs will reduce the morbidity and mortality. VRE surveillance of family members of recently discharged patients VREinfected, to limit the spread of infection.

Key Words- Vancomycin Resistant Enterococci (VRE), MIC-Minimum Inhibitory Concentration, Vancomycin, Teicoplanin, Linezolid, Sensitive, Nosocomial Infection.
\end{abstract}

Citation: Modi G.B., et al. (2012) Prevalenace of Vancomycin Resistant Enterococci in Tertiary Care Hospital, Western, India. International Journal of Microbiology Research, ISSN: 0975-5276 \& E-ISSN:0975-9174, Volume 4, Issue 2, pp.-182-185.

Copyright: Copyright@2012 Modi G.B., et al. This is an open-access article distributed under the terms of the Creative Commons Attribution License, which permits unrestricted use, distribution, and reproduction in any medium, provided the original author and source are credited.

\section{Introduction}

The enterococci have emerged as major causes of nosocomial infections, recognized as the 3rd most common cause of bacteremia. This increase in infection is due in part to resistance to standard therapies, such as high level aminoglycosides and the betalactam antimicrobial agents- and more recently, to the glycopeptides, including vancomycin and teicoplanin. VRE can remain viable in the environment for an extended time period, and therefore poise a problem for infection control in hospitals and nursing homes. In addition, these enterococci have been detected as part of the enteric flora in non-symptomatic patients. These colonized patients serve as potential sources for transfer of this organism to other patients and medical personnel. Enterococci are primarily opportunistic pathogens. Progress in medical technology and intensive use of broad spectrum antibiotics in the hospitals had been responsible for emergence of these organisms as important nosocomial pathogens [1]. Though the major problem in treatment of VRE infection arises in endocarditis, the urinary tract is the commonest site from where bacteraemia can occur. There are very few reports on isolation of VRE from India [2]. It is not always easy to assess the clinical significance of VRE in routine culture or to differentiate colonisation from infection. Therefore, the pre- 
sent study was undertaken to look for vancomycin resistance in enterococci obtained in significant numbers from various clinical samples at a tertiary care centre, Ahmedabad, Gujarat, Western India.

\section{Epidemiology}

During the 1960s and 1970s, it was seen that gram negative organisms were leading causes of hospital acquired infections. Gram-positive organisms were typically sensitive to most antibiotics. However, with the emergence of gram-positive organisms as leading causes of hospital acquired infection in the 1990s, called for a re-evaluation of public research priorities. One of the organisms that caused concern was Vancomycin Resistant Enterococcus (VRE) that was first reported in France in 1988 [3]. Since then, the incidences of Vancomycin Resistant Enterococcus have been reported in many parts of the world. The number of cases infected with VRE, as reported by the US Centers for Disease Control and Prevention, has risen from 99 in 1992 to 278 in 1994. The more recent reports are both distressing as well as alarming. Studies propose that prolonged hospital stay and the irrational use of vancomycin were important risk factors for both vancomycinresistances in Enterococci. Other significant factors that were associated with the emergence of vancomycin-resistant Enterococci were renal dialysis and or renal failure, prior aminoglycoside and the use of third generation cephalosporins. It also emphasized on the importance of screening for VRE in clinical samples. Vancomycin resistant Enterococcus is known to cause significant mortality and morbidity [4]. Enterococcus, or vancomycin-resistant enterococci (VRE), are bacterial strains of the genus Enterococcus that are resistant to the antibiotic vancomycin. To become VRE, Vancomycin-sensitive enterococci typically obtain new DNA in the form of plasmids or transposons which encode genes that confer Vancomycin resistance. This acquired Vancomycin resistance is distinguished from the lower-level, natural Vancomycin resistance of certain enterococcal species including $E$. gallinarum and E. casseliflavus. High-level Vancomycin-resistant E. faecalis and $E$. faecium clinical isolates were first documented in Europe in the late 1980s. Since then, VRE have been associated with outbreaks of hospital-acquired (nosocomial) infections around the world. In the United States, Vancomycin-resistant E. faecium was associated with $4 \%$ of healthcare-associated infections reported to the Centers for Disease Control and Prevention National Healthcare Safety Network from January 2006 to October 2007. VRE can be carried by healthy people who have come into contact with the bacteria. The most likely place where such contact can occur is in a hospital (nosocomial infection) [5].

\section{Materials and Methods}

From July 2010 to July 2011, as per table-1, a total of 250 enterococcal isolates - 81 from urine, 91 from blood, 27 from wound swabs, 18 from pus and 33 from fluids (ascitic fluid 6 , cerebrospinal fluid 17 and peritoneal dialysis fluid 2 and pleural fluid 8 ) were processed. Identification of isolates up to the species level was done by standard biochemical tests according to the conventional scheme of Facklam and Collins [6]. Screening for Vancomycin resistance was done by agar screen methods [7] on MuellerHinton agar (MHA). Antibiotic sensitivity was carried out by Kirby Bauer disc diffusion test [8] as per CLSI guidelines. Susceptibility to Vancomycin was performed by using $30 \mu \mathrm{g}$ disc. Minimum Inhibitory Concentration (MIC) of all the isolates were done by Vancomycin Ezy MICTM Strip ranging from $0.016 \mu \mathrm{g} / \mathrm{ml}$ to $256 \mu \mathrm{g} / \mathrm{ml}$. Plates were incubated at $35^{\circ} \mathrm{C}$ for 24 hours. Susceptibility to high level Gentamicin by using $120 \mu \mathrm{g}$ disc, Penicillin by 10 unit disc and Teicoplanin by using $30 \mu \mathrm{g}$ disc were also performed. Enterococcus faecalis ATCC 29212 and Enterococcus faecalis ATCC 51299 (Vancomycin resistant strain) were used as control strain. The records of the patients whose samples grew VRE in significant numbers were reviewed for demography, clinical findings, underlying medical problems, surgical procedures, invasive devices, and treatment with antimicrobial agents and outcome.

\section{Results}

Out of the 250 Enterococcus species, 58\% (145) were Enterococcus faecalis and $42 \%$ (105) were Enterococcus faecium. 10 isolates were resistant to Vancomycin disc, giving an overall VRE positivity of $4 \%$. Out of $10 \mathrm{VRE}, 40 \%$ (4) were isolated from urine, $40 \%$ (4) from blood and $20 \%$ (2) from wound swabs. Of the 10 patients with VRE, 4 were female, 6 were male [table-3], between age 45 to 70 year [table-2], 4 were isolated from patients of urinary tract infection, 4 from patients of septicemia and 2 from patients of surgical site wound infections [table- 4]. The Vancomycin MIC for these isolates were $8,12 \& 16 \mu \mathrm{g} / \mathrm{ml}$ for three and $256 \mu \mathrm{g} / \mathrm{ml}$ for seven, while the remaining isolates had MIC less than or equal to $4 \mu \mathrm{g} / \mathrm{ml}$. In this study, $1.2 \%$ (3) showed intermediate resistance and $2.8 \%$ (7) were resistant to Vancomycin by MIC according to CLSI guidelines. In this study, among $10 \mathrm{VRE}$ isolates, $80 \%$ (8) were E. faecium and $20 \%$ (2) were E. faecalis. $23.60 \%$ (59) out of 250 isolates were resistant to high level Gentamicin and all VRE were high level aminoglycoside resistants.

Table 2- Age-wise Distribution of Patients with VRE Isolate

\begin{tabular}{|c|c|c|}
\hline $\begin{array}{l}\text { AGE GROUP } \\
\text { (IN YEAR) }\end{array}$ & $\begin{array}{l}\text { NO. OF PATIENTS FROM } \\
\text { ENTEROCOCI WERE } \\
\text { ISOLATED (TOTAL 250) }\end{array}$ & $\begin{array}{l}\text { NO. OF PATIENTS FROM } \\
\text { VRE WERE ISOLATED } \\
\text { (TOTAL 10) }\end{array}$ \\
\hline $0-1$ & $41+1$ & 00 \\
\hline $1-15$ & 19 & 00 \\
\hline $16-30$ & 29 & 00 \\
\hline $31-45$ & 38 & 00 \\
\hline $46-60$ & 47 & $6(12.76 \%)$ \\
\hline $61-75$ & 76 & $4(05.26 \%)$ \\
\hline $76-100$ & 00 & 00 \\
\hline
\end{tabular}

Table 3- Sex Distribution of Patient from Which VRE Were Isolated

\begin{tabular}{|lll|}
\cline { 1 - 1 } SEX & NO. OF PATIENTS FROM & NO. OF PATIENTS FROM \\
& ENTEROCOCI WERE ISO- & $\begin{array}{l}\text { VRE WERE ISOLATED } \\
\text { (TOTAL 10) }\end{array}$ \\
& LATED (TOTAL 250) & $06(04.08 \%)$ \\
MALE & 147 & $04(03.88 \%)$ \\
\hline FEMALE & 103 & \\
\hline
\end{tabular}

All the VRE strains were resistant to Penicillin and Ampicillin. All the isolates of enterococci $(100 \%)$ were sensitive to Linezolid \& Tigecycline including VRE and $97.20 \%$ (243) were sensitive to Teicoplanin. All the isolated E.faecium were sensitive to Streptrogramin including VRE. Out of 10 VRE, 7 were resistant and 3 were sensitive to Teicoplanin, so they were $70 \%$ of VanA and $30 \%$ of VanB phenotype respectively. E.faecium was the commonest amongst VRE. 
Table 4- Prevalence of VRE in Infective Clinical Conditions

\begin{tabular}{lll}
$\begin{array}{l}\text { CLINICAL } \\
\text { CONDITIONS }\end{array}$ & $\begin{array}{l}\text { NO. OF SPECIMEN } \\
\text { FROM ENTEROCOCI } \\
\text { WERE ISOLATED } \\
\text { (TOTAL 250) }\end{array}$ & $\begin{array}{l}\text { NO. OF SPECIMEN } \\
\text { FROM VRE WERE } \\
\text { ISOLATED (TOTAL 10) }\end{array}$ \\
$\begin{array}{l}\text { SEPTICEMIA- } \\
\begin{array}{l}\text { BACTEREMIA } \\
\text { URINARY TRACT }\end{array}\end{array}$ & 91 & $04(04.39 \%)$ \\
INFECTIONS & & $04(04.93 \%)$ \\
$\begin{array}{l}\text { WOUND ABCESS } \\
\text { PUS }\end{array}$ & 27 & $02(07.40 \%)$ \\
INTRA-CAVITARY & 18 & 00 \\
INFECTIONS & 33 & 00 \\
\hline
\end{tabular}

\section{Discussion}

VRE in our hospital is considered as MDRO (Multiple Drug Resistant Organism) \& is notified to clinician as critical /alert. The infection control team takes all the action whenever VRE was detected as per the MDRO management protocol. VRE patient carrier needs to be isolated. All staff needs to be screened. Screening can be done by Stool, rectal, or perirectal swabs and Urine [9]. VRE may inhabit a host and cause no discernable problem that is called colonization. VRE infections may be difficult to cure because the bacteria do not respond to many antibiotics. Sometimes more than one antibiotic is prescribed to help stop the infection. Part of treatment protocol may include sending samples of your blood, urine, or stool to a lab to see if you still have VRE in your body. VRE infection can occur throughout the body with the most common body sites being the urinary tract, surgical wounds, and/or bloodstream [10].

Recently, a phenotypic classification system was devised to categorize the VRE into three groups: vanA strains, which show highlevel Vancomycin resistance (minimum inhibitory concentrations [MIC] of $>32 \mathrm{mcg}$ per $\mathrm{ml}$ ) and resistance to Teicoplanin; vanB strains, which have variable resistance to Vancomycin (MICs of 4 to $>128 \mathrm{mcg}$ per $\mathrm{ml}$ ) and susceptibility to Teicoplanin; and vanC strains, which show intrinsic resistance to low-levels of Vancomycin (MICs of 2 to $16 \mathrm{mcg}$ per $\mathrm{ml}$ ) and susceptibility to Teicoplanin. It is important for the laboratorian to distinguish these strains from the other enterococci which show high-level Vancomycin resistance, since the former are not considered an epidemiological threat for nosocomial transfer and are usually susceptible susceptible to standard therapies.

A major reason for the survival of Enterococcus in hospital environment is their intrinsic resistance to several commonly used antibiotics and, perhaps more important, their ability to acquire resistance to all currently available antibiotics, either by mutation or through the transfer of plasmids and transposons [11]. While Enterococcus is inherently resistant to most of the drugs, Vancomycin resistance complicates treatment and management of the disease. The prognosis is also affected leading to increased mortality and morbidity [12]. The other factors that are also affected are the added burden on costs incurred by the patient in the form of prolonged hospital stay and medication.

Some of the risks for acquiring VRE infection are: persons who have been previously treated with Vancomycin and combinations of other antibiotics, persons who are hospitalized, particularly when they receive antibiotic treatment for long periods of time, persons with weakened immune systems, such as patients in intensive-care units, cancer, or transplant wards, persons who have undergone surgical procedures, such as abdominal or chest surgery, persons with medical devices that stay in for some time, such as urinary catheters or central intravenous catheters. Enterococcal infections are more common in elderly people, particularly those in long-term care facilities and skilled nursing homes because they are more likely to experience infection risk factors, such as exposure to medical instruments. VRE is transmitted from person to person most commonly by healthcare workers whose hands have inadvertently become contaminated, either from feces, urine, or blood of a person carrying the organism. It can also be spread indirectly via hand contact with open wounds or by touching contaminated environmental surfaces, where the bacterium can survive for weeks. VRE is not transmitted through the air [13].

Vancomycin complexes with the d-alanyl-d-alanine terminal of normal peptidoglycan cell wall precursors, thereby inhibiting cell wall synthesis. The genes associated with high-level Vancomycin resistance in enterococci encode a ligase responsible for the synthesis of the depsipeptide d-alanyl-d-lactate. This depsipeptide is incorporated into the terminal portion of the peptidoglycan cell wall precursor, limiting Vancomycin-peptidoglycan precursor binding [14].

Currently, Linezolid is the only oral agent approved by the Food and Drug Administration for treatment of infections caused by VRE. Linezolid inhibits ribosomal protein synthesis but at a different site from other agents that target the ribosome (Chloramphenicol, Macrolides, Lincosamides, Streptogramin, Aminoglycosides, Tetracycline). Consequently, existing mechanisms of resistance to these agents do not confer cross-resistance to Linezolid. Linezolid is the anti-VRE drug used most commonly. Other anti-VRE drugs, Teicoplanin, Tigecycline and Sterptogramin (only for E.faecium) can be used [14].

There is no vaccine to protect against VRE. The most important infection control measure is wash hands before eating, drinking, applying personal care products, and after using the toilet. Bacteria can survive on surfaces like railings, faucets and handles for up to seven days. Routine cleaning of these surfaces with regular household cleaners can also help reduce spread of bacteria. Visitors need to follow hospital guidelines to prevent the spread of VRE. This includes washing hands or using alcohol hand rub when entering and leaving the hospital and/or the patient room. Precautions shall be taken to protect other patients and hospital staff from VRE infection.

\section{Conclusion}

Guidelines have been established by $\mathrm{CDC}$ to prevent the spread of Vancomycin resistance. Each hospital needs to be familiar with the guidelines for the prevention of Vancomycin resistance and establish a policy that reflects their unique needs. The principle recommendations advocated are: 1) the prudent use of Vancomycin; 2) An ongoing education program for all hospital staff about the threat of VRE; 3) A cooperative effort between health care providers and hospital microbiology laboratory personnel that will allow VRE to be promptly and accurately detected; and, 4) The implementation of appropriate infection-control measures to prevent person-to-person spread of VRE;5) screening of health care workers in order to identify carrier rates 6) surveillance cultures in high prevalence areas such as intensive care units and operation 
theatres are immediate requirements in order to keep the spread of Vancomycin resistance under control.

\section{References}

[1] Gold H.S. (2001) Clin Infect Dis, 33, 210-291.

[2] Mathur P., Kapil A., Chandra R., Sharma P., Das B. (2003) Indian J. Med. Res,118, 25-28.

[3] Thalia I. Nicas, Wu C.Y.E., Hobbs J.N., Jr., Preston D.A., Allen N.E. (1989) Antimicrobial Agents and Chemotherapy, 33 (7), 1121-1124.

[4] Suchitra J.B., Lakshmidevi N. (2009) Journal of Microbiology and Antimicrobials., 1(1), 09-11.

[5] en.wikipedia.org/wiki/Vancomycin-resistant_Enterococcus.

[6] Facklam R.R., Collins M.D. (1989) J. Clin. Microbiol., 27, 731740.

[7] Methods for dilution antimicrobial susceptibility tests for bacte- ria that grow aerobically. 5th ed. NCCLS. (2000)

[8] Bauer A.W., Kirby W.M., Sherries J.C., Turck M. (1966) Am. J. Clin. Pathol., 45, 493-496.

[9] Agata D.E.M. (2002) Clin. Infect. Dis. 34, 167-172.

[10]Murray B.E. (1990) Clinical Microbiology Reviews, 3, 46-65.

[11]Vancomycin-Resistant Enterococci, Overview Skip Navigation Department of Health and Human Services, National Institutes of Health.

[12]Mayo Clinic (2006) Proceedings, 81(4), 529-536.

[13]Vancomycin-Resistant Enterococci (2005), HealthLink BC File \#74 HealthLink BC File \#74.

[14]Centers for Disease Control and Prevention (1995) Recommendations for Preventing the Spread of Vancomycin Resistance: Recommendations of the Hospital Infection Control Practices Advisory Committee MMWR, 44(12).

Table 1- Ward Wise Prevalence of Enterococci in Various Clinical Specimens

\begin{tabular}{lllllll} 
WARD/I & BLOOD & URINE & PUS & SWAB & FLUID & TOTAL \\
SPECIMEN & & & & & 00 & $12(04.80 \%)$ \\
ICU & 08 & 03 & 00 & 01 & 14 & $95(38.00 \%)$ \\
MEDICAL WARD & 58 & 21 & 01 & 01 & 13 & $69(27.60 \%)$ \\
$\begin{array}{l}\text { SURGICAL } \\
\text { WARD }\end{array}$ & 14 & 11 & 16 & 15 & 03 & $36(14.40 \%)$ \\
$\begin{array}{l}\text { PAEDIATRIC } \\
\text { WARD }\end{array}$ & 09 & 24 & 00 & 00 & 03 & $32(12.80 \%)$ \\
$\begin{array}{l}\text { GYNECOLOGY } \\
\text { WARD }\end{array}$ & 02 & 18 & 01 & 08 & 00 & $06(02.40 \%)$ \\
OPD & 00 & 04 & 00 & 02 & & $250(100 \%)$ \\
TOTAL & $91(36.40 \%)$ & $81(32.40 \%)$ & $18(07.20 \%)$ & $27(10.80 \%)$ & $33(13.20 \%)$ & 250 \\
\hline
\end{tabular}

